\title{
Application of finite difference method in the study of diffusion with chemical kinetics of first order
}

\author{
Juan Carlos Beltrán-Prieto ${ }^{1, a}$, Karel Kolomazník ${ }^{1}$ \\ ${ }^{1}$ Department of Automation and Control Engineering, Faculty of Applied Informatics, Tomas Bata University in Zlín, nám. T. G. Masaryka \\ 5555, 76001 Zlín, Czech Republic
}

\begin{abstract}
The mathematical modelling of diffusion of a bleaching agent into a porous material is studied in the present paper. Law of mass conservation was applied to analize the mass transfer of a reactant from the bulk into the external surface of a solid geometrically described as a flat plate. After diffusion of the reactant, surface reaction following kinetics of first order was considered to take place. The solution of the differential equation that described the process leaded to an equation that represents the concentration profile in function of distance, porosity and Thiele modulus. The case of interfacial mass resistance is also discused. In this case, finite difference method was used for the solution of the differential equation taking into account the respective boundary conditions. The profile of concentration can be obtained after numerical especification of Thiele modulus and Biot number.
\end{abstract}

\section{Introduction}

Bleaching is a chemical process performed to make a material whiter or lighter [1]. Some of the common bleaching agents used in the industry (i.e. paper coatings, oils, pulp, industrial wastewater, waxes) are sulfur dioxide, calcium hypochlorite, sodium hydrosulfite, hydrogen peroxide or sodium peroxide, ozone, among others) [2]. It is normally carried out by immersing the desired material in a recipient containing the bleaching agent which diffuses through the porous of the solid by random atomic motion.

The governing equation for the mass transfer of this process can be described by the Fick's second law which solution depends on the boundary conditions [3]. The mathematical description of this process is important for the proper quantification of the concentration of the bleaching agent and to understand its effect on the solid surface after the reaction takes place.

\section{Description of the mathematical model}

A microscopic mass balance for the concentration of bleaching agent is performed by considering the accumulation, convective mass transfer and diffusive transfer of bleaching solution through the pores of the solid and the reaction term as expressed in (1). The rate of reaction can be expressed as the disappearance of bleaching agent. Accordingly, it is possible to monitor the decrease in color of the material using a spectrophotometer.

The reaction rate term is considered in the equation because of the existence of an interaction between the

\footnotetext{
$\overline{{ }^{a}}$ Corresponding author: prieto@fai.utb.cz
}

solute and the surface of the solid. Assuming that a first order reaction takes place during the bleaching process, the reaction rate per unit of volume of the solid is described in (2)

$$
\begin{aligned}
\frac{\partial c}{\partial \tau}+V \nabla c & =\nabla(D \nabla c)+R \\
-\frac{\partial c}{\partial \tau} & =k c
\end{aligned}
$$

where $c$ represents the concentration of bleaching agent, $\tau$ the time, $V$ the volume, $\mathrm{D}$ is the diffusivity, and $k$ is the rate constant.

For the purpose of modeling this phenomena, we consider that the diffusion of the well stirred bleaching agent solution occurs in one dimension through a symmetrical flat plate with homogeneous and isotropic properties (the physical and mechanical properties are equal in all orientations of the solid), that the diffusivity is constant (independent of concentration), without interfacial resistance to the mass transport. Accordingly, we consider a steady state situation, in which the concentration will not change in time. Additionally, we suppose the absence of flow of mass due to convection, the equation (1) is transformed into (3) [4]

$$
D \frac{\partial^{2} c}{\partial x^{2}}-k c=0
$$

The previous equation describes that the concentration $(c)$ is a function of position $(x)$. It is a linear partial differential equation (parabolic) that can be solved for a given settings (initial and boundary conditions)[5]. This is a two-point boundary value problem, therefore the 
concentration needs to be specified at both $\mathrm{x}=0$ and $\mathrm{x}=\mathrm{L}$, as described in equations (4) and (5)

$$
\begin{aligned}
& x=0, \frac{d c}{d x}=0 \\
& x=L, c=\varepsilon \cdot c_{L}
\end{aligned}
$$

The first condition expresses that at the center of the solid there is no diffusion flux and therefore express a condition of symmetry. The second condition describes the case of diffusion in a well-stirred media, in which the concentration on the surface of the solid(c) is equal to the concentration of the bulk $\mathrm{c}_{\mathrm{L}}$ with the proper consideration of the porosity of the solid $(\varepsilon)$.

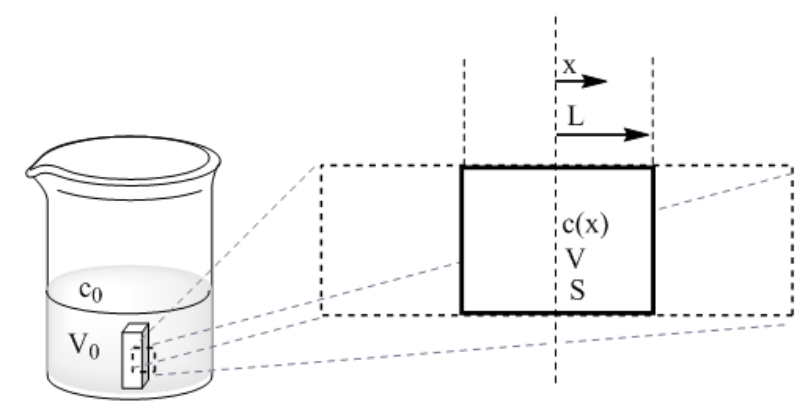

Figure 1. Geometric representation of the system under study.

For the solution of the problem, the mass balance equation and boundary conditions are expressed in dimensionless form using the parameters described in (6):

$$
X=\frac{x}{L}, C=\frac{c}{\varepsilon \cdot c_{L}}
$$

As a result: $\mathrm{x}=\mathrm{X} \cdot \mathrm{L}, \mathrm{c}=\mathrm{C} \cdot \varepsilon \cdot \mathrm{C}_{\mathrm{L}}, \mathrm{dx}=\mathrm{L} \cdot \mathrm{dX}, \mathrm{dc}=\varepsilon \cdot$ $\mathrm{c}_{\mathrm{L}} \cdot \mathrm{dC}$, and $\frac{d^{2} c}{d x^{2}}=\frac{d}{L d X}\left[\frac{\varepsilon \cdot c_{L} \cdot d C}{L d X}\right]$, therefore we obtain (7)

$$
\frac{d^{2} c}{d x^{2}}=\frac{\varepsilon \cdot c_{L} \cdot d^{2} C}{L^{2} d X^{2}}
$$

Substitution of (7) and previous dependences into (3) leads to (8):

$$
\frac{d^{2} C}{d X^{2}}-\frac{k L^{2}}{D} C=0
$$

from where we can define the dimensionless parameter of Thiele module $(\varphi)$, which represents the ratio of the rate of reaction to the rate of diffusion [6] as expressed in (9).

$$
\varphi^{2}=\frac{k L^{2}}{D}
$$

The boundary conditions in dimensionless values adopt the form of the next equations:

$$
\begin{aligned}
& X=0, \frac{d C}{d X}=0 \\
& X=1, C=1
\end{aligned}
$$

Equation (8) has the form of the characteristic equation $\mathrm{m}^{2}-\varphi^{2}=0$ giving a set of conjugate roots $\mathrm{m}_{1}=\varphi$ and $\mathrm{m}_{2}=-\varphi$. As a result, the general solution of the differential equation is (12)

$$
C=c_{1} e^{\varphi X}+c_{2} e^{-\varphi X}
$$

For the purpose of applying boundary conditions, it is often convenient to represent equation (12) using hyperbolic functions. As a result, equation (12) is transformed into (13)

$$
C=c_{1} \cosh \varphi X+c_{2} \sinh \varphi X
$$

The value of the constants $\mathrm{C}_{1}$ and $\mathrm{C}_{2}$ can be determined taking into considerations the boundary equations (10) and (11). As a result $C_{1}=\left(1-C_{2} \sinh \varphi\right) / \cosh \varphi$ and $C_{2}=0$. Substitution of the previous values in equation (12) leads to (14) (expressed in the original variables)

$$
c=\frac{\varepsilon \cdot c_{L} \cdot \cosh \varphi_{L}^{x}}{\cosh \varphi}
$$

This equation allows us to obtain the concentration profile $(c)$ as a function of distance $(x)$ for a given values of porosity as represented in Figure 1 and for given values of Thiele modulus.

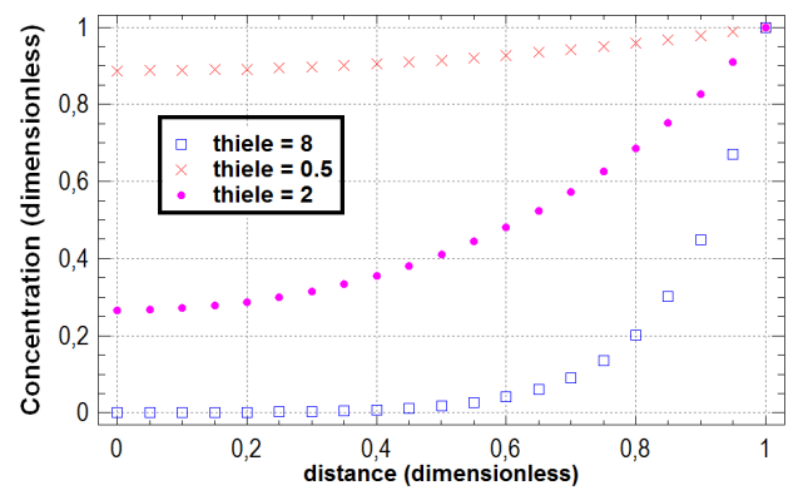

Figure 2. Plot of dimensionless concentration profile as a function of distance for different Thiele values $(\square: 0.8, \mathrm{x}: 0.5$, and $\bullet: 2)$.

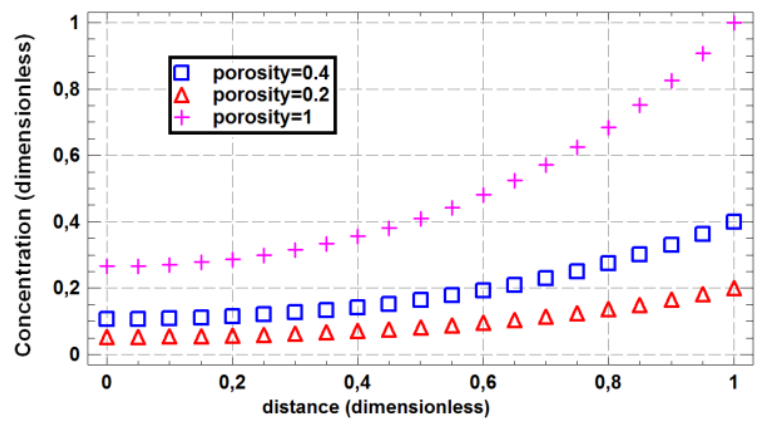

Figure 3. Plot of dimensionless concentration profile as a function of distance using different values of porosity $(\square: 0.4, \Delta$ $: 0.2$ and $+: 1)$. 
Following we proceed to determine the effectiveness factor $(\eta)$, which indicates the ratio between the reaction rate and the reaction rate without resistance to diffusion [7], [8] as described in (15)

$\eta=\frac{-r}{-r_{S}}=\frac{\text { actual reaction rate }}{\text { reaction rate without diffusion }}=\left.\frac{1}{\varphi^{2}} \frac{d C}{d X}\right|_{X=1}$

Solving equation (14) leads to equation (16)

$\eta=\left.\frac{1}{\varphi^{2}}\left(\frac{1}{\cosh \varphi}(\varphi \cdot \sinh \varphi X)\right)\right|_{X=1}=\frac{1}{\varphi}\left(\frac{\sinh \varphi}{\cosh \varphi}\right)=\frac{\tanh \varphi}{\varphi}$

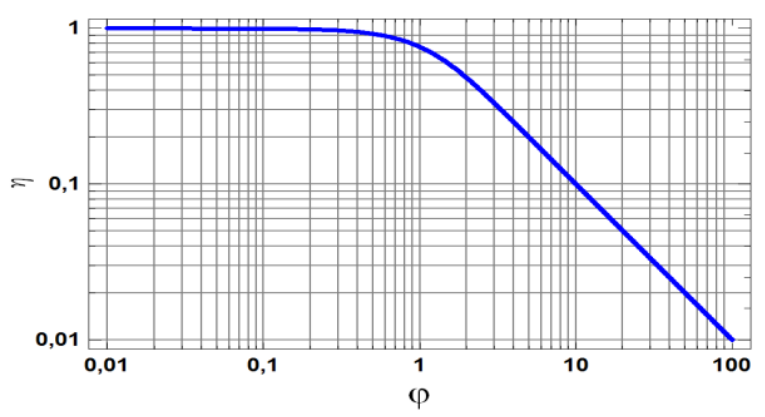

Figure 4. Plot of effectiveness factor as a function of Thiele modulus in logarithmic scale.

The reaction rate without diffusion is also the reaction rate at the surface of the solid, therefore the overall reaction rate is calculated by multiplying the intrinisic reaction rate per unit volume and the effectiveness factor as described in equation (17) and finally in (18)

$$
\begin{gathered}
r=\eta \cdot r_{s}=\eta \cdot k \cdot c \\
r=\frac{\tanh \varphi}{\varphi} \cdot k \cdot c=\frac{\tanh \sqrt{\frac{k L^{2}}{D}}}{\sqrt{\frac{k L^{2}}{D}}} k \cdot c
\end{gathered}
$$

In the case that there is interfacial mass resistance, then the boundary condition is given by $x=0, \frac{d c}{d x}=0$; and $x=L,-D \frac{d c}{d x}=k_{s} \cdot \varepsilon\left(c-c_{L}\right)$ where $k_{s}$ represents the mass transfer coefficient. Accordingly, the same dimensionless parameters still apply and the boundary conditions are transformed in:

$X=0, \frac{d C}{d X}=0 ; X=1, \frac{d C}{d X}=-\frac{L \cdot k_{s}}{D}(C \cdot \varepsilon-1)$

Where the quantity $-L \cdot k_{s} / D$ is also known as the mass transfer Biot number $\mathrm{Bi}_{\mathrm{m}}$ which represents the ratio of internal diffusion resistance to external convection resistance [9]. In this case, equation (8) with boundary conditions expressed in (19) can be solved using finite difference method [10]. In this case, we used 4 interior nodes

$$
\begin{gathered}
\text { Node } 1 \rightarrow \frac{c_{2}-c_{1}}{\Delta X}=0 \\
\text { Node } 2 \rightarrow \frac{c_{3}-2 c_{2}+c_{1}}{\Delta X^{2}}-\varphi^{2} c_{2}=0 \\
\text { Node } 3 \rightarrow \frac{c_{4}-2 c_{3}+c_{2}}{\Delta X^{2}}-\varphi^{2} c_{3}=0 \\
\text { Node } 4 \rightarrow \frac{c_{5}-2 c_{4}+c_{3}}{\Delta X^{2}}-\varphi^{2} c_{4}=0 \\
\text { Node } 5 \rightarrow \frac{c_{6}-2 c_{5}+c_{4}}{\Delta X^{2}}-\varphi^{2} c_{5}=0 \\
\text { Node } 6 \rightarrow \frac{c_{6}-c_{5}}{\Delta X}=B i_{m}(C \cdot \varepsilon-1)
\end{gathered}
$$

$\Delta \mathrm{X}$ is calculated from equation (26)

$$
\Delta X=1 /(n+1)
$$

Where $\mathrm{n}$ is the number of interior nodes, therefore $\Delta \mathrm{X}$ is 0.2 . Equations (20) to (26) results in a system of linear equations represented in equation (27)

$$
\begin{aligned}
& \left(\begin{array}{cccccc}
-5 & 5 & 0 & 0 & 0 & 0 \\
25 & -50-\varphi^{2} & 25 & 0 & 0 & 0 \\
0 & 25 & -50-\varphi^{2} & 25 & 0 & 0 \\
0 & 0 & 25 & -50-\varphi^{2} & 25 & 0 \\
0 & 0 & 0 & 25 & -50-\varphi^{2} & 25 \\
0 & 0 & 0 & 0 & 5 & -5-B i_{m}
\end{array}\right)\left(\begin{array}{l}
C_{1} \\
C_{2} \\
C_{3} \\
C_{4} \\
C_{5} \\
C_{6}
\end{array}\right)= \\
& \left(\begin{array}{c}
0 \\
0 \\
0 \\
0 \\
0 \\
-B i_{m}
\end{array}\right)
\end{aligned}
$$

The solutions of the previous system of equations leads to find the profile of concentration after numerical especification of Thiele modulus and Biot number. For numerical simulation we consider porosity equal to 1 and values of $\varphi^{2}=4$, and $B i_{m}=16$ and $\varphi^{2}=8$, and $B i_{m}=0.4$. This is represented in Figure 5 .

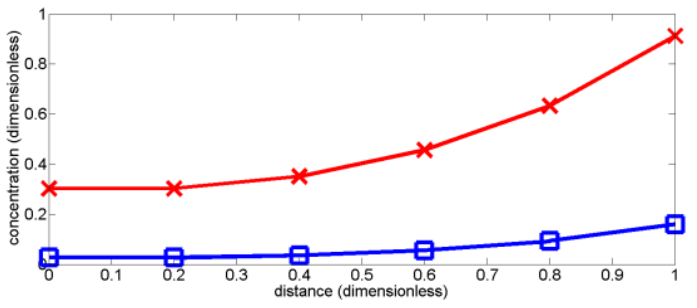

Figure 5. Plot of concentration profile for two cases. Case 1: $\varphi^{2}=4, B i_{m}=16$ (cross) and case $2: \varphi^{2}=8$, $B i_{m}=0.4$ (square).

\section{Conclusions}

The study of diffusion of a liquid into a porous material with a first order reaction taking place has been studied. In order to determine the effect of mass transfer on the overall reaction rate, an effectiveness factor was determined. This factor is also important to describe how far the reactant diffuses into the solid before the reaction takes place. 
When the values of $\eta$ are near to 1 , the reaction is surface reaction limited but if this value is small $(\eta<<1)$ the reaction is diffusion limited. If Thiele modulus is large, internal diffusion limits the overall rate of reaction, but if it is small, surface reaction is rate limiting. The case of boundary condition with interfacial resistance was studied. This is present in the case that the bulk fluid moves slowly. Finite difference method was used for the solution of a problem describing the mass transport and reaction with this boundary condition.

\section{Acknowledgements}

This work was supported by the Ministry of Education, Youth and Sports of the Czech Republic within the National Sustainability Programme project No. LO1303 (MSMT-7778/2014).

\section{References}

1. International Food Information Service, IFIS Dictionary of Food Science and Technology
(International Food Information Service, Wiley Blackwell, Reading, 2009).

2. M. Ash, I. Ash, Handbook of Paper and Pulp Chemicals (Synapse Information Resources, Inc., New York, 2013).

3. R. B. Bird, W. E. Stewart, E. N. Lightfoot, Transport Phenomena (John Wiley and Sons, Inc, New York, 2002).

4. J. Welty, C. Wicks, R. Wilson, Fundamentals of Momentum, heat, and Mass Transfer, (John Wiley and Sons, Mexico, 2008).

5. Y. A. Cengel, Heat And Mass Transfer: A Practical Approach (McGraw-Hill, New York, 2006).

6. I. Tosun, Modeling in Transport Phenomena (Elsevier, The Netherlands, 2007).

7. B. Godongwana, PloS One, 11, 1-13 (2016)

8. G. A. Adagiri, G. Babagana, A. A. Susu, Int. J. Res. Rev, Applied. Sci. 13, 716-739 (2012).

9. R. G. Rice, D. D. Do, Applied mathematics and modeling for chemical engineers, (John Wiley \& Sons, Inc, New Jersey, 2012).

10. R. L. Burden, J. D. Faires, Numerical Analysis (Brooks Cole, United Kingdom, 2002). 